\title{
Flexible sigmoidoscopy in colorectal cancer screening: implications of different colonoscopy referral strategies
}

\author{
Tobias Niedermaier ${ }^{1,2} \cdot$ Korbinian Weigl $^{1,2,4} \cdot$ Michael Hoffmeister ${ }^{1} \cdot$ Hermann Brenner ${ }^{1,3,4}$
}

Received: 3 November 2017 / Accepted: 3 May 2018/Published online: 12 May 2018

(C) The Author(s) 2018

\begin{abstract}
Flexible sigmoidoscopy (FS) screening reduces colorectal cancer incidence and mortality. Its potential to detect proximal neoplasms depends on colonoscopy referral. We estimated diagnostic performance of sigmoidoscopy using 12 different referral criteria in detecting colorectal cancer and advanced adenomas. Colonoscopy results from 14,947 participants of screening colonoscopy in Germany were used to derive sensitivity of sigmoidoscopy for colorectal cancer, advanced adenomas (AAs), and any advanced neoplasms in the proximal colon. It was assumed that FS detects the same neoplasms as colonoscopy within its reach and that distal neoplasms would be followed by colonoscopy. In addition, numbers of colonoscopies needed (NCN) to detect one proximal advanced neoplasm were calculated. The most advanced findings during colonoscopy were colorectal cancer in 213 subjects (1.4\%), AA in 1539 subjects $(10.2 \%)$ and non-advanced adenomas in 2988 subjects (19.8\%). Without colonoscopy referral, overall sensitivities for any colorectal cancer, advanced adenoma and any advanced neoplasm (proximal or distal) would be 79, 65 and 66\%, respectively. These sensitivities could be increased to up to 86,83 and $84 \%$ by the referral strategies investigated. Compared to referral due to advanced adenomas, referral due to non-advanced adenomas would substantially increase the NCN at a modest gain in sensitivity. Sensitivities were higher and NCNs were lower in men than in women for every strategy. In conclusion, colonoscopy referral can substantially increase sensitivity of sigmoidoscopy-based screening, but the gain by referral due to nonadvanced adenomas substantially increases NCN compared to referral due to advanced neoplasms only. Major sex differences may call for sex-specific referral strategies.
\end{abstract}

Keywords Colorectal neoplasia $\cdot$ Detection $\cdot$ Screening $\cdot$ Colonoscopy referral

\begin{abstract}
Abbreviations
AA Advanced adenoma

AN Advanced neoplasm

CI Confidence interval

CRC Colorectal cancer

FS Flexible sigmoidoscopy

NCN Number of colonoscopies needed
\end{abstract}

Electronic supplementary material The online version of this article (https://doi.org/10.1007/s10654-018-0404-x) contains supplementary material, which is available to authorized users.

Hermann Brenner

h.brenner@dkfz.de

1 Division of Clinical Epidemiology and Aging Research, German Cancer Research Center (DKFZ), Im Neuenheimer Feld 581, 69120 Heidelberg, Germany

2 Medical Faculty Heidelberg, University of Heidelberg, Heidelberg, Germany

\section{Introduction}

Colorectal cancer (CRC) is a common, yet largely preventable disease. Although colonoscopy is the gold standard in CRC and advanced adenoma detection, disadvantages such as higher costs, discomfort and complication rates as well as lower adherence limit its use as primary screening method. Flexible sigmoidoscopy (FS) reliably detects colonic neoplasms in the distal colon and

3 Division of Preventive Oncology, German Cancer Research Center (DKFZ) and National Center for Tumor Diseases (NCT), Heidelberg, Germany

4 German Cancer Consortium (DKTK), German Cancer Research Center (DKFZ), Heidelberg, Germany 
rectum, which is where the majority of all colorectal neoplasms occur [1]. Proximal neoplasms can be detected by FS only indirectly if detection of distal lesions is followed by colonoscopy, as recommended in CRC screening guidelines [2-4]. Several randomized clinical trials (RCTs) demonstrated that FS screening reduces CRC incidence and mortality [5-8]. FS screening is offered in Italy, UK and in the USA [9].

The RCTs on effectiveness of FS screening differed in their colonoscopy referral criteria [5-8]. While the UK Flexible Sigmoidoscopy Trial [10] imposed the highest thresholds for colonoscopy referral, less restrictive criteria were imposed in the SCORE trial (Italy) [5], the PLCO trial (USA) [6] and the NORCCAP trial (Norway) [11]. Accordingly, colonoscopy referral rates and detection rates of proximal colonic neoplasms differed between these trials [12].

More restrictive referral strategies imply lower detection rates than less restrictive criteria, but also lower numbers of colonoscopies needed $(\mathrm{NCN})$ to detect one relevant proximal finding [12]. Comparative evaluations of various potential colonoscopy referral strategies within the same study population are sparse [13-15]. Differential performance of the same strategies in men and women can be expected due to the typically higher prevalence of colorectal neoplasia among men. In Germany, FS is not routinely conducted for primary CRC screening, despite proven effectiveness and lower costs and effort compared to colonoscopy screening. We used colonoscopy findings in a large German CRC screening population to compare the expected impact on diagnostic performance (sensitivities and $\mathrm{NCN}$ ) of 12 referral strategies following screening FS in men and women.

\section{Materials and methods}

\section{Study design and study population}

We used data from the ongoing KolosSal study, which has been described elsewhere [16, 17]. In this statewide cohort study, initiated in 2005 in Saarland, a small state (1 million inhabitants) in southwestern Germany, CRC incidence and mortality are monitored among participants of screening colonoscopy. For our analysis, we used baseline data from participants recruited in 33 gastroenterology practices in Saarland from January 2, 2006, through October 31, 2012.

In the German screening colonoscopy program, subjects aged $\geq 55$ years (no upper age limit) are eligible for screening colonoscopy, with the option of a second screening colonoscopy $\geq 10$ years later. Almost all screening colonoscopies are conducted in practices of gastroenterology or internal medicine. To become eligible, endoscopists must have conducted $\geq 200$ colonoscopies and $\geq 50$ polypectomies under supervision in the preceding two calendar years. To maintain eligibility, endoscopists must conduct $\geq 200$ colonoscopies per year and $\geq 10$ polypectomies per year. Histopathologic examination of removed polyps is performed decentrally; endoscopists send polyps to a certified pathological laboratory of their choice.

Nearly all practices conducting screening colonoscopies in Saarland agreed to recruit patients for the study cohort. Eligible patients had to be residents of Saarland undergoing screening colonoscopy in a participating practice. 18,997 subjects were recruited between January 2, 2006, and October 31, 2012. The study was approved by ethics committees of the University of Heidelberg and of the Medical Association of Saarland. Each participant provided written informed consent.

Representativeness of our results for an average-risk screening population was ensured by excluding participants matching any of the following criteria (Fig. 1): $<55$ or $\geq 80$ years of age $(\mathrm{N}=703)$, history of CRC or inflammatory bowel disease $(\mathrm{N}=275)$; colonoscopy in the preceding 5 years $(\mathrm{N}=1692)$. To minimize the number of screening colonoscopies with missed neoplasms, subjects with inadequate bowel preparation before colonoscopy $(\mathrm{N}=1216)$ or incomplete colonoscopy (coecum not reached; $\mathrm{N}=164$ ) were excluded. Thus, 14,947 participants were retained for the analysis. Approximately one fourth of them $(23.5 \%, \mathrm{~N}=3499)$ had a previous colonoscopy more than 5 years ago.

\section{Data collection}

Participants were recruited in the practices prior to screening colonoscopy, typically at a preparatory visit. They provided basic information on CRC risk and preventive factors in a standardized questionnaire and agreed that copies of colonoscopy and histology reports were forwarded by the physician for this study. Those reports were reviewed by trained investigators who were blinded with respect to questionnaire data. Participants were classified into the following categories according to the most advanced finding at colonoscopy: CRC, advanced adenoma (AA), nonadvanced adenoma, other. Adenomas with at least one of the following features were defined as AAs: size $\geq 1 \mathrm{~cm}$, tubulo-villous or villous components, highgrade dysplasia.

\section{Statistical analyses}

Sensitivity of FS was derived from colonoscopy results. FS was assumed to detect the same neoplasms as colonoscopy within its reach. Proximal and distal location of neoplasms 


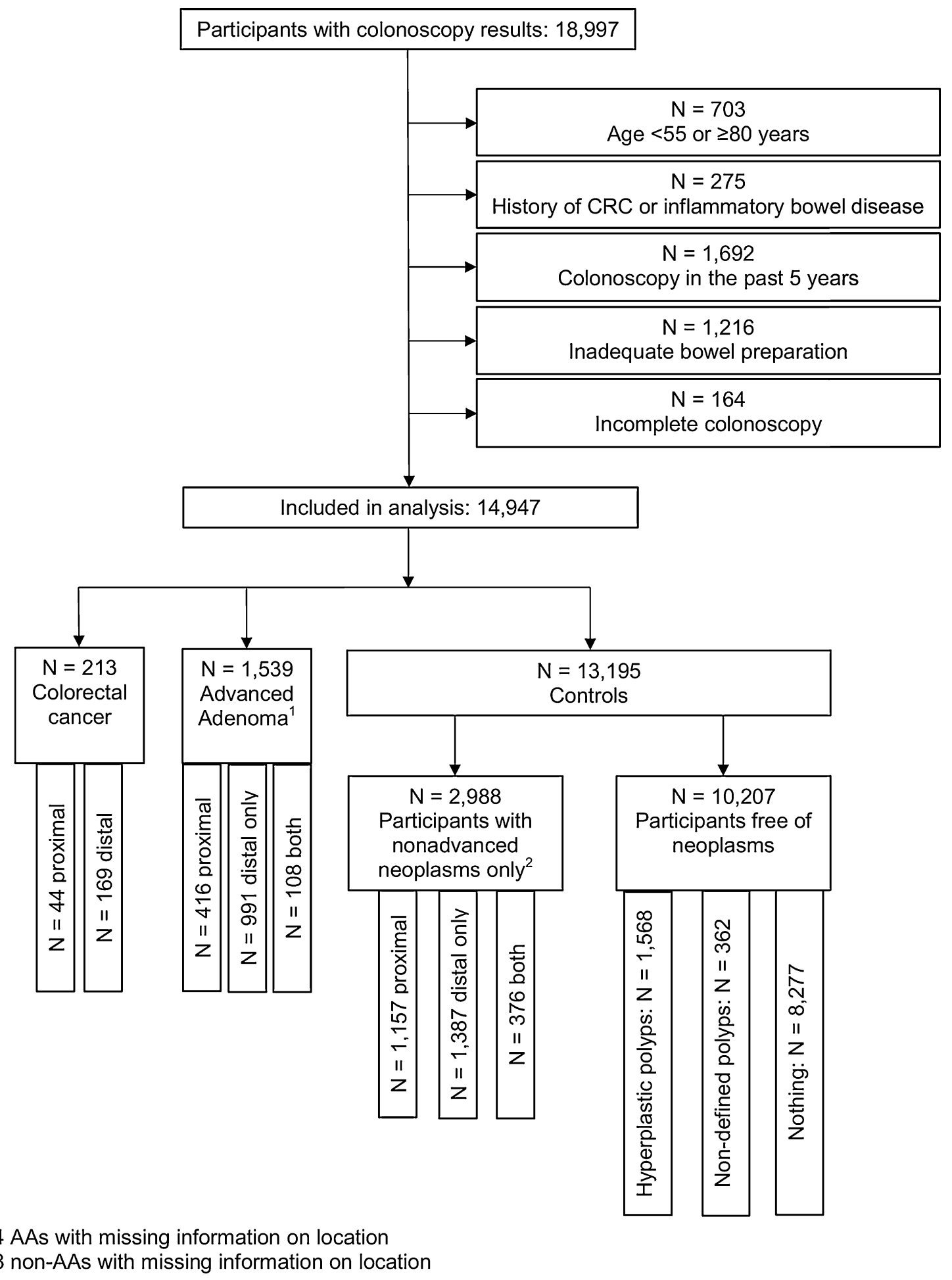

Fig. 1 Flow diagram of the participants in the KolosSal study included in this analysis

were defined according to the assumed reach of FS. In the main analyses, colonoscopic findings were defined as proximal (FS-unreachable) when located proximal to the descending colon and distal otherwise. In sensitivity analyses, findings located proximal to the sigmoid colon were defined as proximal, otherwise as distal. In addition, expected detection of proximal neoplasms due to different referral to colonoscopy after detection of distal neoplasms was examined. 
We investigated the referral criteria of the FS screening trials (UK, SCORE, NORCCAP, US/PLCO, see Table 1) and further recommended or conceivable referral strategies. These strategies used the following referral criteria (based on distal findings) and were sorted according to the number of colonoscopy referrals: $\geq 2$ neoplasms, thereof $\geq 1$ advanced neoplasm (AN, defined as CRC or AA); $\geq 2$ neoplasms; $\geq 1$ histology-defined AN (high-grade dysplasia, tubulo-villous components or both); $\mathrm{AN} \geq 1 \mathrm{~cm}$; any AN; any neoplasm; any neoplasm or hyperplastic polyp (defined as such by the pathological labs, excluding adenomatous polyps, serrated polyps, pseudopolyps and other findings).

\section{Outcomes}

Overall sensitivities for detecting (proximal or distal) CRC, AAs or any AN were investigated as outcomes for the aforementioned referral criteria. Sensitivities were calculated as the number of subjects with AN correctly identified by FS itself or colonoscopy referral divided by the total number of subjects with AN detected during colonoscopy.

In addition, we calculated the NCN per "FS-unreachable" (proximal) outcome (CRC, AA, any AN). This number equals the number of participants with a neoplasm in the distal colon or rectum that would lead to follow-up colonoscopy (which differs between the investigated strategies), divided by the number of participants in whom a proximal AN would be detected by follow-up colonoscopy. To investigate the burden and benefit of relaxing referral criteria, we calculated the number of additional colonoscopies needed to detect one additional proximal AN when comparing more extensive to the most restrictive examined referral strategy ("incremental NCN"). Finally, negative predictive values were calculated for all outcomes and referral strategies, i.e., the probability of a subject that would not be referred to colonoscopy having no proximal AN.

All outcome measures (sensitivities, NCN) were calculated stratified by gender and for the entire study population.

Statistical analyses were performed in $\mathrm{R}$ [18] version 3.2.5. For sensitivities, 95\% Clopper-Pearson (binomial) confidence intervals (CIs) were calculated using the $\mathrm{R}$ package "binom" [19] version 1.1-1.

Table 1 Investigated colonoscopy referral criteria according to findings at flexible sigmoidoscopy

\begin{tabular}{|c|c|c|}
\hline Referral criteria & Details & Ref. \\
\hline \multicolumn{3}{|l|}{ Trials } \\
\hline UK FS screening trial & $\begin{array}{l}\text { CRC, one distal polyp or adenoma }>1 \mathrm{~cm} \text {, (tubulo-)villous histology, } \\
\text { HGD, } \geq 3 \text { adenomas or } \geq 20 \text { hyperplastic polyps above the rectum }\end{array}$ & {$[10]$} \\
\hline SCORE & $\begin{array}{l}\text { Distal polyp(s) }>5 \mathrm{~mm} \text {, (tubulo-)villous histology, HGD, } \geq 3 \text { adenomas or } \\
\text { CRC }\end{array}$ & [5] \\
\hline NORCCAP & $\mathrm{CRC}$, one distal polyp $\geq 1 \mathrm{~cm}$ or any adenoma & {$[11]$} \\
\hline US PLCO trial & $\begin{array}{l}\text { Score } \geq 4 \text { [age }(50-54: 0,55-59: 1,60-64: 2,65-70: 3)+\text { gender (female: } \\
\text { 0, male: } 1)+ \text { most advanced distal finding (no polyps: } 0 \text {, hyperplasia: } 1, \\
\text { tubular adenoma }<10 \mathrm{~mm}: 2 \text {, advanced lesion (tubular } \\
\text { adenoma } \geq 10 \mathrm{~mm}, \text { villous histology, HGD, CRC: } 3 \text { )] }\end{array}$ & {$[6]$} \\
\hline \multicolumn{3}{|l|}{ Other } \\
\hline$\geq 2$ neoplasms, $\geq 1$ AN & $\begin{array}{l}\text { At least two distal adenomas, thereof at least one advanced adenoma, or } \\
\text { CRC }\end{array}$ & \\
\hline$\geq 2$ neoplasms & At least two distal adenomas or CRC & \\
\hline Histology-defined AN & Distal (tubulo-)villous adenoma or HGD or CRC & \\
\hline$A N \geq 1 \mathrm{~cm}$ & Distal large $(\geq 1 \mathrm{~cm})$ adenoma or $C R C$ & \\
\hline Any AN & Any distal advanced adenoma or CRC & \\
\hline Any neoplasm & Any distal adenoma or CRC; Recommended by several guidelines ${ }^{\mathrm{a}}$ & {$[2-4]$} \\
\hline Any neoplasm or HPP & Any distal adenoma or hyperplastic polyp or CRC & \\
\hline
\end{tabular}




\section{Results}

\section{Study population}

The study population comprised 14,947 subjects. $49.0 \%$ were male. Mean age was 63.2 years, 213 subjects (1.4\%) had CRC, 1539 (10.3\%) had AAs and 2988 (20.0\%) had non-advanced adenomas as the most advanced findings at screening colonoscopy (Table 2). Prevalences of CRC and AA were approximately twice as high in men as in women.

\section{Diagnostic performance of FS}

Expected diagnostic performance of FS, assuming different colonoscopy referral strategies following FS reaching the descending colon, is summarized in Table 3. Consistently lower sensitivities were observed in women than in men. Sensitivity for CRC without any colonoscopy referral was estimated as $84 \%$ (95\% CI 77-90\%) in men and 70\% (95\% CI $58-80 \%$ ) in women. $67 \%$ of AAs in men and $63 \%$ of AAs in women were within the reach of FS. In men and women, a steady increase in sensitivities was observed when assuming colonoscopy referral and applying gradually less restrictive referral criteria. Up to $85 \%$ of AN would have been detected in men and up to $76 \%$ in women, assuming referral due to any neoplasm or hyperplastic polyp. All sensitivities were lower by approximately 5 percentage points when assuming that FS does not reach and visualize the descending colon (Supplementary Table 1).

The SCORE criteria would have performed similarly to the UK criteria. Among both sexes combined, approximately one out of six AN missed by the UK criteria would have been detected using the NORCCAP criteria (sensitivities: 75 and 79\%, respectively). Due to the high number of colonoscopies in men, PLCO criteria would have had the highest overall sensitivity for AN (85\%) and the by far largest number of colonoscopies (5229 compared to between 1421 and 3220 for the other trials' criteria). In women, the NORCCAP criteria would have achieved higher sensitivities for AN (73\%) than the PLCO criteria (72\%), but would also have required more colonoscopies (1216 vs. 1062). The differences in sensitivities for AN between different referral strategies were larger in men than in women. Gender differences in sensitivity within the same strategy were most pronounced in the US (PLCO) criteria (20\%-points for any AN), which consider age and sex in addition to colonoscopic findings. In all other strategies, differences were between 7 and 10\%-points.

Comparing referral criteria used in FS trials and further conceivable strategies, including the widely recommended criterion "any distal neoplasm" [2-4], a similar range of overall and sex-specific sensitivities and numbers of colonoscopies would be expected. With most criteria, the majority of advanced proximal neoplasms, 54-88\%, would still be expected to be missed (Table 4). The US criteria were the only criteria to detect more than half of all proximal ANs (55\%). This was driven by the high sensitivity among men $(75 \%)$, whereas more than three out of four AN would still have been missed in women (sensitivity 23\%). Overall, similar patterns emerged when assuming that FS reaches the sigmoid colon only (Supplementary Table 1). For CRC, sensitivities were 1-5\%points lower. For AA and any AN, 3-6\%-points lower sensitivities were achievable.
Table 2 Characteristics of the KolosSal study population

\begin{tabular}{|c|c|c|c|c|c|c|}
\hline \multirow[t]{2}{*}{ Characteristic } & \multicolumn{2}{|c|}{ Total, $\mathrm{N}=14,947$} & \multicolumn{2}{|c|}{ Men, $N=7323$} & \multicolumn{2}{|c|}{ Women, $\mathrm{N}=7624$} \\
\hline & $\mathrm{N}$ & $\%$ & $\mathrm{~N}$ & $\%$ & $\mathrm{~N}$ & $\%$ \\
\hline \multicolumn{7}{|l|}{ Age (years) } \\
\hline $55-59$ & 5672 & 37.9 & 2680 & 36.6 & 2992 & 39.2 \\
\hline $60-64$ & 3263 & 21.8 & 1608 & 22.0 & 1655 & 21.7 \\
\hline $65-69$ & 3049 & 20.4 & 1527 & 20.9 & 1522 & 20.0 \\
\hline $70-74$ & 2077 & 13.9 & 1045 & 14.3 & 1032 & 13.5 \\
\hline $75-79$ & 886 & 5.9 & 463 & 6.3 & 423 & 5.5 \\
\hline \multicolumn{7}{|c|}{ Most advanced finding at screening colonoscopy } \\
\hline Colorectal cancer & 213 & 1.4 & 140 & 1.9 & 73 & 1.0 \\
\hline Advanced adenoma & $1539^{\mathrm{a}}$ & 10.3 & 985 & 13.5 & 554 & 7.3 \\
\hline Non-advanced adenoma & 2988 & 20.0 & 1721 & 23.5 & 1267 & 16.6 \\
\hline Hyperplastic polyps & 1568 & 10.5 & 796 & 10.9 & 772 & 10.1 \\
\hline Other/unspecified polyps & 362 & 2.4 & 192 & 2.6 & 170 & 2.2 \\
\hline No finding & 8277 & 55.4 & 3489 & 47.6 & 4788 & 62.8 \\
\hline
\end{tabular}

${ }^{a}$ Thereof 24 AA cases with missing information on location, leaving 1515 AA cases for analyses 
Table 3 Expected overall sensitivities in \% (95\% CIs) of flexible sigmoidoscopy (FS) based screening with different colonoscopy referral strategies in the male and female KolosSal study population, sorted by the number of colonoscopies conducted

\begin{tabular}{|c|c|c|c|c|c|c|c|c|}
\hline \multirow[t]{3}{*}{ Sex } & \multirow{3}{*}{$\begin{array}{l}\text { Colonoscopy referral } \\
\text { criterion after FS screening }\end{array}$} & \multirow{3}{*}{$\begin{array}{l}\text { Number of } \\
\text { colonoscopies }\end{array}$} & \multicolumn{6}{|c|}{ Most advanced finding at colonoscopy } \\
\hline & & & \multicolumn{2}{|c|}{$\mathrm{CRC}(\mathrm{N}=140 / 73)$} & \multicolumn{2}{|c|}{ AA $(\mathrm{N}=971 / 544)$} & \multicolumn{2}{|c|}{ Any AN (N = 1111/617) } \\
\hline & & & $\begin{array}{l}\mathrm{N} \\
\text { detected }\end{array}$ & $\begin{array}{l}\text { Sensitivity [\%] } \\
(95 \% \mathrm{CI})\end{array}$ & $\begin{array}{l}\mathrm{N} \\
\text { detected }\end{array}$ & $\begin{array}{l}\text { Sensitivity [\%] } \\
(95 \% \mathrm{CI})\end{array}$ & $\begin{array}{l}\mathrm{N} \\
\text { detected }^{\mathrm{b}}\end{array}$ & $\begin{array}{l}\text { Sensitivity [\%] } \\
(95 \% \mathrm{CI})\end{array}$ \\
\hline \multirow[t]{12}{*}{ Men } & No referral & 0 & 118 & $84(77-90)$ & 646 & $67(63-69)$ & 752 & $68(65-70)$ \\
\hline & UK FS screening trial & 965 & 126 & $90(84-94)$ & 740 & $76(73-79)$ & 866 & $78(75-80)$ \\
\hline & SCORE & 1146 & 126 & $90(84-94)$ & 751 & $77(75-80)$ & 877 & $79(76-81)$ \\
\hline & NORCCAP & 2004 & 127 & $91(85-95)$ & 787 & $81(78-83)$ & 914 & $82(80-84)$ \\
\hline & US (PLCO) & 4167 & 135 & $96(92-99)$ & 887 & $91(89-93)$ & 1022 & $92(90-94)$ \\
\hline & $\geq 2$ neoplasms, $\geq 1$ AN & 395 & 122 & $87(80-92)$ & 686 & $71(68-73)$ & 808 & $73(70-75)$ \\
\hline & $\geq 2$ neoplasms & 630 & 123 & $88(81-93)$ & 703 & $72(69-75)$ & 826 & $74(72-77)$ \\
\hline & Histology-defined $A N^{a}$ & 617 & 124 & $89(82-93)$ & 703 & $72(69-75)$ & 827 & $74(72-77)$ \\
\hline & $A N>1 \mathrm{~cm}$ & 639 & 122 & $87(80-92)$ & 706 & $73(70-75)$ & 828 & $75(72-77)$ \\
\hline & Any AN & 854 & 125 & 89 (83-94) & 729 & $75(72-78)$ & 854 & $77(74-79)$ \\
\hline & Any neoplasm & 1941 & 127 & $91(85-95)$ & 781 & $80(78-83)$ & 908 & $82(79-84)$ \\
\hline & Any neoplasm or HPP & 2737 & 127 & $91(85-95)$ & 816 & $84(82-86)$ & 943 & $85(83-87)$ \\
\hline \multirow[t]{12}{*}{ Women } & No referral & 0 & 51 & $70(58-80)$ & 345 & $63(59-67)$ & 395 & $64(60-68)$ \\
\hline & UK FS screening trial & 456 & 52 & $71(59-81)$ & 372 & $68(64-72)$ & 424 & $69(65-72)$ \\
\hline & SCORE & 586 & 52 & $71(59-81)$ & 377 & $69(65-73)$ & 429 & $70(66-73)$ \\
\hline & US (PLCO) & 1062 & 57 & $78(67-87)$ & 388 & $71(67-75)$ & 445 & $72(68-76)$ \\
\hline & NORCCAP & 1216 & 57 & $78(67-87)$ & 396 & $73(69-76)$ & 453 & $73(70-77)$ \\
\hline & $\geq 2$ neoplasms, $\geq 1 \mathrm{AN}$ & 152 & 52 & $71(59-81)$ & 354 & $65(61-69)$ & 406 & $66(62-70)$ \\
\hline & $\geq 2$ neoplasms & 263 & 53 & $73(61-82)$ & 358 & $66(62-70)$ & 411 & $67(63-70)$ \\
\hline & Histology-defined $A N^{a}$ & 307 & 52 & $71(59-81)$ & 363 & $67(63-71)$ & 415 & $67(63-71)$ \\
\hline & $A N>1 \mathrm{~cm}$ & 314 & 52 & $71(59-81)$ & 359 & $66(62-70)$ & 411 & $67(63-70)$ \\
\hline & Any AN & 422 & 52 & $71(59-81)$ & 370 & $68(64-72)$ & 422 & $68(65-72)$ \\
\hline & Any neoplasm & 1186 & 57 & $78(67-87)$ & 394 & $72(68-76)$ & 451 & $73(69-77)$ \\
\hline & Any neoplasm or HPP & 1874 & 57 & $78(67-87)$ & 412 & $76(72-79)$ & 469 & $76(72-79)$ \\
\hline \multirow{12}{*}{$\begin{array}{l}\text { Both } \\
\text { sexes }\end{array}$} & No referral & 0 & 169 & $79(73-85)$ & 991 & $65(63-68)$ & 1147 & $66(64-69)$ \\
\hline & UK FS screening trial & 1421 & 178 & $84(78-88)$ & 1112 & $73(71-76)$ & 1290 & $75(73-77)$ \\
\hline & SCORE & 1732 & 178 & $84(78-88)$ & 1128 & $74(72-77)$ & 1306 & $76(73-78)$ \\
\hline & NORCCAP & 3220 & 184 & $86(81-91)$ & 1183 & $78(76-80)$ & 1367 & $79(77-81)$ \\
\hline & US (PLCO) & 5229 & 192 & $90(85-94)$ & 1275 & $84(82-86)$ & 1467 & $85(83-87)$ \\
\hline & $\geq 2$ neoplasms, $\geq 1$ AN & 547 & 174 & $82(76-87)$ & 1040 & $69(66-71)$ & 1214 & $70(68-72)$ \\
\hline & $\geq 2$ neoplasms & 893 & 176 & $83(77-87)$ & 1061 & $70(68-72)$ & 1237 & $72(69-74)$ \\
\hline & Histology-defined $A N^{a}$ & 924 & 176 & $83(77-87)$ & 1066 & $70(68-73)$ & 1242 & $72(70-74)$ \\
\hline & $A N>1 \mathrm{~cm}$ & 953 & 174 & $82(76-87)$ & 1065 & $70(68-73)$ & 1239 & $72(70-74)$ \\
\hline & Any AN & 1276 & 177 & $83(77-88)$ & 1099 & $73(70-75)$ & 1276 & $74(72-76)$ \\
\hline & Any neoplasm & 3127 & 184 & $86(81-91)$ & 1175 & $78(75-80)$ & 1359 & $79(77-81)$ \\
\hline & Any neoplasm or HPP & 4611 & 184 & $86(81-91)$ & 1228 & $81(79-83)$ & 1412 & $82(80-84)$ \\
\hline
\end{tabular}

Main analysis assuming that FS reaches and visualizes descending colon

Strategies in italic would not automatically refer subjects with any distal AN to colonoscopy

$C R C$ colorectal cancer, $A A$ advanced adenoma, $A N$ advanced neoplasia, $H P P$ hyperplastic polyp

${ }^{a}$ Histology-defined AN: high-grade dysplasia, (tubulo-)villous histology, CRC, or any combination thereof

${ }^{\mathrm{b}}$ This number refers to participants in whom all proximal and distal AN are detected. It is smaller than the sum of participants with CRC or AA detected as their most advanced finding in case of no referral, because those detected with distal CRC may still have proximal AA that would not be detected in case of no referral 
As a consequence of the relatively low prevalence of proximal AN in the study population $(581 / 14,947=3.9 \%)$, subjects not referred to colonoscopy were unlikely to have proximal AN, despite the low sensitivities of the strategies for their detection: Even without colonoscopy referral, the negative predictive value (NPV) for AN was approximately $95 \%$, increasing to approximately $97 \%$ when using more comprehensive referral criteria. NPVs for the endpoint CRC were very close to $100 \%$ for all strategies (Supplementary Table 3). Assuming a more limited reach of FS did not change the results materially (Supplementary Table 4).

\section{Number of colonoscopies per neoplasm detected}

From the numbers of colonoscopy referrals and detected proximal AN with each strategy, we calculated the average NCN to detect one proximal AN. As shown in Table 4, $5-57 \%$ of men and $2-25 \%$ of women would need to undergo colonoscopy. The UK and SCORE strategies, referring approximately one out of seven men and one out of 13 women to colonoscopy, would detect approximately one out of three proximal ANs in men and one out of seven proximal ANs in women each. Applying the NORCCAP and US criteria would detect nearly one out of two and three out of four proximal ANs in men, respectively, but require colonoscopy follow-up in $27 \%$ (NORCCAP) and $57 \%$ (US) of all men. While only $16 \%$ (NORCCAP) and $14 \%$ (US) of women would undergo colonoscopy with these strategies, detection of proximal ANs would also remain very limited (only 26 and $23 \%$, respectively).

The NCNs increase gradually with more extensive referral strategies and are substantially higher in strategies with colonoscopy referral after detection of any neoplasm than in those with referral after detection of advanced neoplasms only. The most restrictive strategy examined assumed colonoscopy referral only due to CRC or at least two detected distal neoplasms, requiring one of them to be advanced. That strategy had the lowest NCN among both, men and women: Only 4\% of the study population $(\mathrm{N}=547)$ would undergo colonoscopy and 67/581 $=12 \%$ of all proximal AN would be detected. Compared to this strategy, requiring $547 / 67=8.2$ colonoscopies to detect one proximal AN, a steep increase in the numbers of colonoscopies required to detect an additional AN was observable for all other strategies. This increase was larger with more extensive referral criteria. For example, taking both sexes together, colonoscopy referral due to any AN required 729 additional colonoscopies (1276-547) and detected 62 additional AN (129-67), resulting in an incremental NCN of 729/62 = 11.8. By comparison, referral due to any distal neoplasm would have required 2580 additional colonoscopies for 145 additionally detected AN. The incremental NCN of $17.8(2580 / 145)$ was thereby considerably higher than for the "any AN" referral strategy.

For all strategies, NCN were substantially lower for men than for women. In men, between 7.1 and 15.4 colonoscopies would be conducted per proximal AN detected, assuming FS to visualize the descending colon, whereas between 13.8 and 25.3 colonoscopies would be conducted per proximal AN detected in women. Assuming FS to reach the sigmoid colon only, all NCNs would be slightly lower among men, whereas both slightly higher and slightly lower NCNs were estimated for women (Supplementary Table 2).

\section{Discussion}

We estimated diagnostic performance of a once-only FS for the detection of advanced colorectal neoplasms by modeling different colonoscopy referral criteria following FS in a German CRC screening population. Without colonoscopy referral, assuming that the descending colon is not reached, FS would detect $62 \%$ of $\mathrm{AN}$ in men and $59 \%$ of AN in women. At the upper end, with FS reaching the entire descending colon and colonoscopy referral following the US (PLCO) criteria, 57\% of all men and would undergo colonoscopy, yielding sensitivities of $92 \%$ for AN. In women, colonoscopy following any neoplasm or hyperplastic polyp would yield the highest number of colonoscopies (25\% of all women) and NCN (25.3) and achieve $76 \%$ sensitivity for AN. Compared to referral due to distal AN only (NCN 15.6), the number of colonoscopies to detect one proximal AN would strongly increase, and a large share of this increase in sensitivity could be achieved with much lower colonoscopy referral rates using less extensive referral strategies.

We found major sex differences in sensitivity (higher in men) and the NCN to detect one proximal AN (higher in women) that might suggest considering sex-specific referral strategies. Although sensitivity could be increased among women by using less restrictive referral strategies compared to men, this would further aggravate the gender discrepancies in the NCN. For example, assuming FS to reach and visualize the descending colon, referral of women with any distal neoplasm or HPP would approximately yield the same overall sensitivity for detecting any AN (76\%) as referral of men with any distal advanced neoplasm only $(77 \%)$ (see Table 3$)$. However, such an approach would require 25.3 colonoscopies to detect one additional proximal AN among women, three times the corresponding number among men (8.4, see Table 4$)$. To achieve a better use of colonoscopy resources, quite a contrary approach may make sense: Referring men to colonoscopy after detecting any distal neoplasm, but 


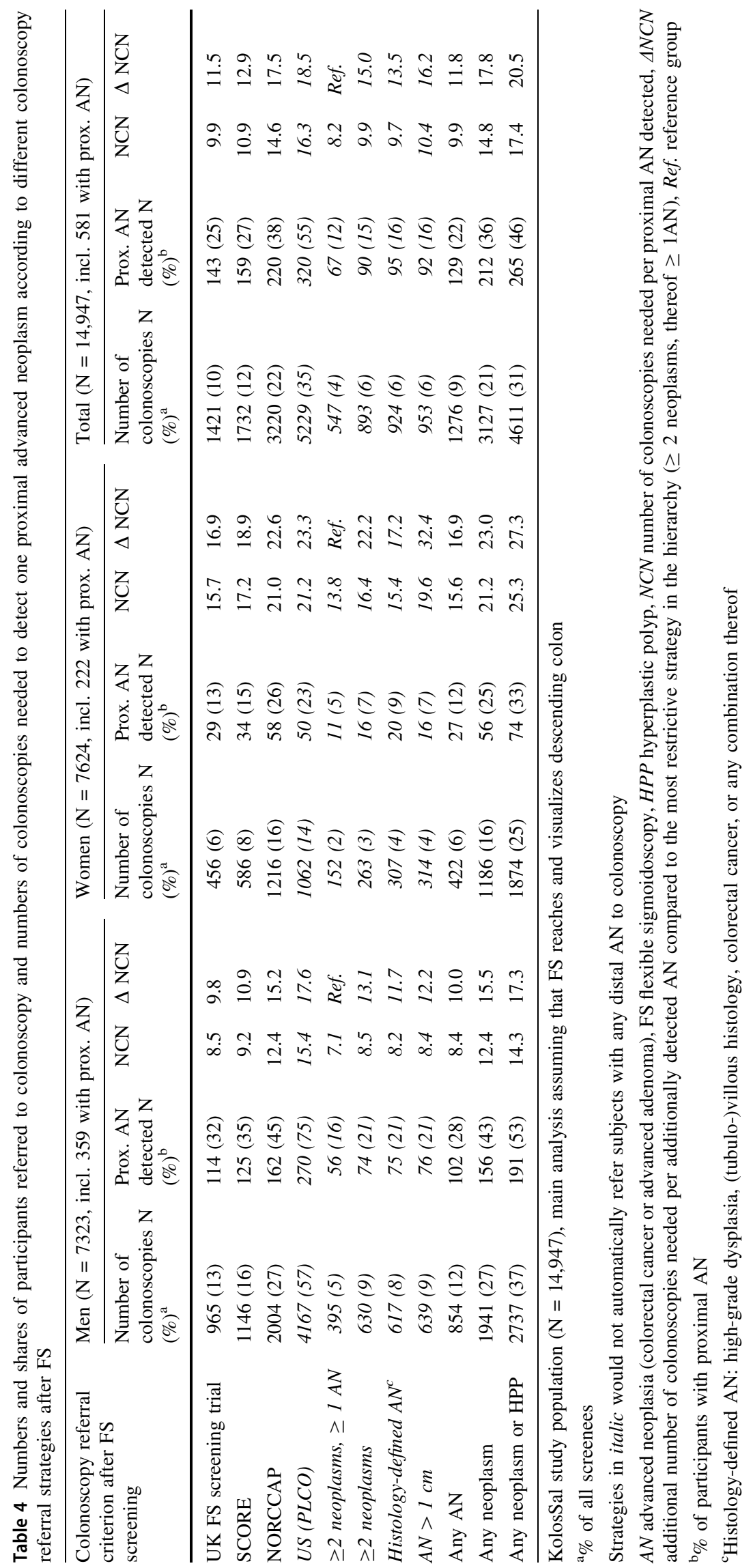


women only after detecting a distal AN would not only result in quite comparable NCN for both sexes (12.4 and 15.6, respectively, see Table 4), but also lead to a higher number of detected proximal AN $(156+27=183$ vs. $102+74=176)$, despite an overall much lower number of colonoscopies $\quad(1941+422=2363 \quad$ vs. $854+1874=2728)$ compared to the aforementioned "equal sensitivity" scenario.

Several earlier studies had reported an increased risk of proximal $\mathrm{AN}$ in the presence of specific distal findings [20-22], supporting the use of colonoscopy referral strategies based on such findings, but only few studies (one each from Japan, Spain and China) have explicitly compared expected performance of various referral strategies in terms of detection of proximal AN [13-15]. Like our study, they reported increased sensitivity for proximal AN with more extensive referral strategies, and two of them [14, 15] also reported major sex differences. Castells et al. [14] obtained virtually identical sensitivities for proximal AN using the UK, SCORE and NORCCAP criteria (22, 31 and $37 \%$, respectively). Similar to our study, sensitivities were consistently higher in men than in women. The US strategy, where the largest sex-specific differences could be expected, was not examined, though. In the study of Wong et al. [15], estimated achievable sensitivities for proximal AN using the SCORE and NORCCAP strategies were very similar to those obtained in our study, with 31 and $38 \%$, respectively. Compared to our study, they found lower sensitivities when using the UK criteria (14 vs. $25 \%$ ) or the US criteria (42 vs. 55\%). Our study expands the evidence from these previous studies in several important respects, in that we included a much larger number of potential referral strategies (12 compared to 2, 3 or 4 ), along with a larger number of participants with proximal AN (581 compared to 319, 212, and 132, respectively). Furthermore, we provided, for the first time, detailed sex specific analyses for different assumptions regarding the reach of FS. The mean age of the so far largest study population from Japan (48 years) had been below the typical age at which FS is recommended and conducted, and this study had assessed only two referral strategies (any distal AN versus any distal neoplasm) for both sexes combined [13]. Our study is the first to explicitly quantify the "incremental NCN", i.e., the number of additional colonoscopies needed to detect one additional proximal AN when comparing more extensive to more restrictive referral strategies. Such "incremental NCN", which we reported for comparisons of referral strategies to the most restrictive strategy under investigation could be derived analogously for comparisons between any other pairs of more restrictive and more comprehensive referral strategy from the data presented in this paper and may be a parameter of particular relevance for delineating the study population that should be referred.
In contrast to a previous study indicating that histologydefined AAs discovered during FS are stronger predictors for proximal AN than large distal adenomas [21], we did not find pronounced differences in sensitivity when using different definitions of AA for referral strategies. However, referral based on size-defined AN tended to perform worse in terms of NCN and $\triangle \mathrm{NCN}$ than referral based on histology-defined AN. Compared to other primary colonoscopy screening populations [23-27], the CRC detection rate was somewhat higher in our study (1.4\%). Similarly, estimated sensitivities of FS for AN detection were mostly higher than previously estimated from meta-analysis results [28]. Possible reasons for the relatively high CRC detection rate include the substantially older age of the study population compared to other studies, conduction of the study in a high incidence country and a high incidence region within Germany [29], and exclusion of subjects with a previous colonoscopy in the past 5 years who have very low CRC detection rates [17]. Despite stringent in- and exclusion criteria applied in our study, it cannot be ruled out entirely that some subjects who underwent screening colonoscopy had other symptoms that motivated them to visit a gastroenterology practice. However, our study thereby accurately reflects the setting in which subjects are recruited in the German colonoscopy screening program. This self-selection might have further contributed to the somewhat higher CRC prevalence in our study. One consequence are consistently lower NCNs than those found in a previous study [27].

Via adenoma removal during FS, a share of CRCs can actually be prevented. Compared to screening colonoscopy, some proximal neoplasms are inevitably missed, thus limiting the preventive potential of FS-only screening. Results from the UK Flexible Sigmoidoscopy Screening Trial with its comparably restrictive referral criteria indicate that even a single FS can achieve a significant and long-lasting reduction of distal CRC incidence and mortality [8]. Reductions of proximal colon cancer incidence were not seen, suggesting that much higher colonoscopy referral rates would be needed to have a significant effect on proximal colon cancer incidence. Whether expanding the colonoscopy referral rates would be the best way to enhance sensitivity and effectiveness of FS based screening appears questionable in the light of our results. Other approaches, such as conduction of a single FIT first, using a positive FIT result as referral criterion to colonoscopy, followed by a once-only FS in FIT negatives [28, 30] are potentially more promising. In the NORCCAP study, CRC incidence and mortality of FS screening alone were also compared to a combination with FIT [7]. The study found no significant differences in detection rates of adenomas or CRC and statistically non-significant reductions in CRC mortality in both groups. Incidence was higher and 
mortality lower in the group with combined FIT and FS screening compared to sole FS screening. In a previous approach of modeling performance of FS, alone and combined with FIT [28], no individual patient data was available and thus, performance of FS could not be examined for different referral strategies. Estimated overall sensitivities of FS for AN detection were 60, 68 and 72\%, assuming colonoscopy referral rates of 0,20 and $30 \%$, respectively. In the present study, estimated sensitivities for AN were somewhat higher, with 66, 74 and $79 \%$ at comparable referral rates $(0,22$ and $36 \%$ using no referral, any AN or any neoplasm as referral criteria, respectively). Another recent study [30] investigated performance of a single FS alone and combined with FIT for the widely recommended referral criterion due to any distal neoplasm. With sensitivities of $86 \%$ and $72 \%$ of FS alone for CRC and AA, respectively, estimated accuracy of FS was similar to that of the present study. Additional conduction of FIT was estimated to increase sensitivities for CRC to $100 \%$ and sensitivities for AA to at least $72 \%$ and up to $82 \%$, depending on the FIT cutoff.

Our study has several strengths. To our knowledge, it is the most comprehensive analysis of colonoscopy referral criteria, examining 12 different criteria in a very large population of participants of screening colonoscopy. It is the first to explicitly quantify the incremental benefit and burden of extending a relatively restrictive colonoscopy referral strategy following FS. Sensitivities for proximal and for any AN were investigated. All included participants underwent full colonoscopy, ensuring that sensitivities of FS are not overestimated due to missed proximal neoplasms at an incomplete colonoscopy. Participants underwent colonoscopy for primary screening, not for clarification of symptoms. Thus, potential overestimation of sensitivity of FS if symptomatic subjects with a presumably higher prevalence of FS-reachable findings had been included should have been avoided. To our knowledge, our study is the by far largest of its kind, with more than 15,000 participants, including over 1500 AA and 200 CRC cases. For all participants, detailed colonoscopy data were available, including location, size and histopathologic features of every finding. These comprehensive data allowed us to provide precise gender-specific estimates of sensitivity, NCN and the NPV for a range of FS-based screening strategies. Assuming a more limited reach of FS did not alter any of the results materially.

Our study also has limitations. Sigmoidoscopy results were derived from colonoscopy. Although a common approach in studies investigating sensitivity of FS [13-15, 21, 22], performance of FS might differ from that of colonoscopy, e.g. due to different bowel preparation procedures (enema vs. oral bowel cleansing) or the absence of sedation. Evidence in this matter is inconclusive, however [31]. On the other hand, the more convenient preparation procedure of FS might yield higher participation rates which might offset a somewhat smaller sensitivity in the distal colon and rectum compared to colonoscopy. Furthermore, also colonoscopy may miss neoplasms, mainly in the proximal colon. Participants with incomplete colonoscopy $(0.9 \%, \mathrm{~N}=164 / 19,261)$ were excluded from our analyses. Not reaching the coecum will slightly diminish sensitivity of colonoscopy in screening practice. Another factor potentially influencing sensitivity of FS is conduction of the procedure by non-gastroenterologists. Although FS, in contrast to colonoscopy, is frequently conducted by general practitioners and may even by conducted by nurses [32], detection rates are expected to be similar to those of gastroenterologists $[33,34]$. Nevertheless, a certain fraction of FS exams will not be completed in practice, e.g. due to pain or insufficient bowel preparation. Those factors might reduce insertion depth and thereby sensitivity of FS somewhat, although our sensitivity analyses indicated that results were very similar even when only the rectum and sigmoid colon were assumed to be reached and visualized by FS. Full compliance to follow-up colonoscopy due to the examined referral criteria was assumed. Partial non-adherence would reduce the gain in achievable sensitivities compared to FS without colonoscopy referral. For example, assuming only $75 \%$ adherence rate to colonoscopy follow-up after recommended referral would reduce the 24 percentage points increase in sensitivity for AN achievable by applying the US criteria to men to approximately 18 percentage points. In some of the investigated strategies, we assumed that distal AN discovered during FS would be removed immediately without colonoscopy follow-up. Although advanced adenomas can in principle be removed during FS, the American Society of Gastrointestinal Endoscopists (ASGE) recommends polypectomy during FS only after adequate bowel preparation [35]. Finally, our analyses did not consider sessile serrated polyps which were not systematically detected and recorded by many endoscopists during the earlier years of study recruitment.

In conclusion, FS applied with a moderately restrictive colonoscopy referral strategy, such as referral only due to advanced distal findings, would likely achieve high sensitivities for detection of ANs in women, and even higher sensitivities in men, in a large German CRC screening population and require considerably fewer colonoscopies than primary screening colonoscopy. Even for moderately restrictive referral strategies, the share of false-negatives, i.e. of missing a proximal AA or even proximal colon cancer if FS is not followed by colonoscopy, was very low in our study. However, for any referral strategy, much higher numbers of colonoscopies to detect one proximal AN would be needed for women than for men, and these 
numbers would substantially increase with increasingly extensive referral strategies. Restricting colonoscopy referral to those with any advanced distal neoplasm rather than the commonly recommended referral of those with any distal neoplasm, at least among women, should be considered. A drawback of such a strategy would be a potentially higher number of interval cancers. On the other hand, even a single FIT detects the majority of proximal colon cancers and a significant proportion of proximal advanced adenomas [28, 36]. To avoid an increase in the number of interval cancer cases, additional conduction of FIT prior to FS, with colonoscopy referral of FIT-positives and conduction of FS among FIT-negatives might be an alternative.

Acknowledgements We gratefully acknowledge the excellent cooperation of gastroenterology practices and clinics in patient recruitment. We also gratefully acknowledge Isabel Lerch and Dr. Utz Benscheid for their contribution in data collection, monitoring and documentation. Guarantor of the article: Tobias Niedermaier.

Funding The KolosSal study was supported by grants from the Central Research Institute of Ambulatory Health Care in Germany, Berlin, Germany and the German Cancer Aid $(108,230,110,553)$. The sponsors had no role in the study design; in the collection, analysis, and interpretation of data; preparation; review; or approval of the manuscript.

Author contributions HB designed the study. TN conducted the statistical analyses and drafted the manuscript. $\mathrm{HB}, \mathrm{MH}$ and $\mathrm{KW}$ contributed to important intellectual content and critically revised the manuscript. All authors approved the final draft submitted.

Ethical approval The study was approved by ethics committees of the University of Heidelberg and of the Medical Association of Saarland. All procedures performed in studies involving human participants were in accordance with the ethical standards of the institution or practice at which the studies were conducted. Each participant provided written informed consent.

\section{Compliance with ethical standards}

Conflict of interest The authors declare that they have no conflict of interest.

Open Access This article is distributed under the terms of the Creative Commons Attribution 4.0 International License (http://creative commons.org/licenses/by/4.0/), which permits unrestricted use, distribution, and reproduction in any medium, provided you give appropriate credit to the original author(s) and the source, provide a link to the Creative Commons license, and indicate if changes were made.

\section{References}

1. Siegel RL, Miller KD, Fedewa SA, Ahnen DJ, Meester RGS, Barzi A, et al. Colorectal cancer statistics, 2017. CA Cancer J Clin. 2017;67(3):177-93. https://doi.org/10.3322/caac.21395.
2. American Cancer Society. American Cancer Society recommendations for colorectal cancer early detection. American Cancer Society. 2016. http://www.cancer.org/cancer/colonan drectumcancer/moreinformation/colonandrectumcancerearly detection/colorectal-cancer-early-detection-acs-recommenda tions. Accessed Feb 132017.

3. Group Health Cooperative. Colorectal cancer screening guideline. https://www.ghc.org/static/pdf/public/guidelines/colon.pdf. Accessed Apr 132017.

4. Levin B, Lieberman DA, McFarland B, Smith RA, Brooks D, Andrews KS, et al. Screening and surveillance for the early detection of colorectal cancer and adenomatous polyps, 2008: a joint guideline from the American Cancer Society, the US MultiSociety Task Force on Colorectal Cancer, and the American College of Radiology. CA Cancer J Clin. 2008;58(3):130-60. https://doi.org/10.3322/CA.2007.0018.

5. Segnan N, Armaroli P, Bonelli L, Risio M, Sciallero S, Zappa M, et al. Once-only sigmoidoscopy in colorectal cancer screening: follow-up findings of the Italian Randomized Controlled TrialSCORE. J Natl Cancer Inst. 2011;103(17):1310-22. https://doi. org/10.1093/jnci/djr284.

6. Schoen RE, Pinsky PF, Weissfeld JL, Yokochi LA, Church T, Laiyemo AO, et al. Colorectal-cancer incidence and mortality with screening flexible sigmoidoscopy. N Engl J Med. 2012; 366(25):2345-57. https://doi.org/10.1056/NEJMoa1114635.

7. Holme O, Loberg M, Kalager M, Bretthauer M, Hernan MA, Aas $\mathrm{E}$, et al. Effect of flexible sigmoidoscopy screening on colorectal cancer incidence and mortality: a randomized clinical trial. JAMA. 2014;312(6):606-15. https://doi.org/10.1001/jama.2014. 8266.

8. Atkin W, Wooldrage K, Parkin DM, Kralj-Hans I, MacRae E, Shah U, et al. Long term effects of once-only flexible sigmoidoscopy screening after 17 years of follow-up: the UK Flexible Sigmoidoscopy Screening randomised controlled trial. Lancet. 2017;389(10076):1299-311. https://doi.org/10.1016/S01406736(17)30396-3.

9. Vleugels JL, van Lanschot MC, Dekker E. Colorectal cancer screening by colonoscopy: putting it into perspective. Dig Endosc. 2016;28(3):250-9. https://doi.org/10.1111/den.12533.

10. Atkin W, Edwards R, Kralj-Hans I, Wooldrage K, Hart AR, Northover JM, et al. Once-only flexible sigmoidoscopy screening in prevention of colorectal cancer: a multicentre randomised controlled trial. Lancet. 2010;375(9726):1624-33. https://doi.org/ 10.1016/S0140-6736(10)60551-X.

11. Gondal G, Grotmol T, Hofstad B, Bretthauer M, Eide TJ, Hoff G. The Norwegian Colorectal Cancer Prevention (NORCCAP) screening study: baseline findings and implementations for clinical work-up in age groups 50-64 years. Scand J Gastroenterol. 2003;38(6):635-42.

12. Schoen RE, Machicado JD. Detection of advanced neoplasia with FIT versus flexible sigmoidoscopy versus colonoscopy: more is more. Dig Dis Sci. 2015;60(5):1123-5. https://doi.org/10.1007/ s10620-015-3583-2.

13. Kato J, Morikawa T, Kuriyama M, Yamaji Y, Wada R, Mitsushima $\mathrm{T}$, et al. Combination of sigmoidoscopy and a fecal immunochemical test to detect proximal colon neoplasia. Clin Gastroenterol Hepatol. 2009;7(12):1341-6. https://doi.org/10. 1016/j.cgh.2009.04.025.

14. Castells A, Bessa X, Quintero E, Bujanda L, Cubiella J, Salas D, et al. Risk of advanced proximal neoplasms according to distal colorectal findings: comparison of sigmoidoscopy-based strategies. J Natl Cancer Inst. 2013;105(12):878-86. https://doi.org/10. 1093/jnci/djt117.

15. Wong MC, Ching JY, Ng SC, Wong S, Chan VC, Shum JP, et al. Prediction of proximal advanced neoplasia: a comparison of four existing sigmoidoscopy-based strategies in a Chinese population. 
Gut. 2015;64(5):776-83. https://doi.org/10.1136/gutjnl-2014308002.

16. Brenner H, Hoffmeister M, Arndt V, Stegmaier C, Altenhofen L, Haug U. Protection from right- and left-sided colorectal neoplasms after colonoscopy: population-based study. J Natl Cancer Inst. 2010;102(2):89-95. https://doi.org/10.1093/jnci/djp436.

17. Brenner H, Haug U, Arndt V, Stegmaier C, Altenhofen L, Hoffmeister M. Low risk of colorectal cancer and advanced adenomas more than 10 years after negative colonoscopy. Gastroenterology. 2010;138(3):870-6. https://doi.org/10.1053/j.gas tro.2009.10.054.

18. R Core Team. R: a language and environment for statistical computing. R Foundation for Statistical Computing. Vienna, Austria. 2016. http://www.R-project.org/. Accessed 13 Feb 2017.

19. Dorai-Raj S. binom: Binomial confidence intervals for several parameterizations. URL http://cran.r-project.org/web/packages/ binom/index.html. 2014.

20. Gondal G, Grotmol T, Hofstad B, Bretthauer M, Eide TJ, Hoff G. Grading of distal colorectal adenomas as predictors for proximal colonic neoplasia and choice of endoscope in population screening: experience from the Norwegian Colorectal Cancer Prevention study (NORCCAP). Gut. 2003;52(3):398-403. https:// doi.org/10.1136/gut.52.3.398.

21. Levin TR, Palitz A, Grossman S, Conell C, Finkler L, Ackerson L, et al. Predicting advanced proximal colonic neoplasia with screening sigmoidoscopy. JAMA. 1999;281(17):1611-7. https:// doi.org/10.1001/jama.281.17.1611.

22. Imperiale TF, Wagner DR, Lin CY, Larkin GN, Rogge JD, Ransohoff DF. Risk of advanced proximal neoplasms in asymptomatic adults according to the distal colorectal findings. N Engl J Med. 2000;343(3):169-74. https://doi.org/10.1056/ NEJM200007203430302.

23. Morikawa T, Kato J, Yamaji Y, Wada R, Mitsushima T, Shiratori Y. A comparison of the immunochemical fecal occult blood test and total colonoscopy in the asymptomatic population. Gastroenterology. 2005;129(2):422-8. https://doi.org/10.1016/j.gas tro.2005.05.056.

24. Nakazato MYH, Matsushita H, Sato K, Fujita K, Yamanaka Y, Imai Y. Immunologic fecal occult blood test for colorectal cancer screening. Jpn Med Assoc J. 2006;49(5/6):203-7.

25. Park DI, Ryu S, Kim YH, Lee SH, Lee CK, Eun CS, et al. Comparison of guaiac-based and quantitative immunochemical fecal occult blood testing in a population at average risk undergoing colorectal cancer screening. Am J Gastroenterol. 2010;105(9):2017-25. https://doi.org/10.1038/ajg.2010.179.

26. Chiu HM, Lee YC, Tu CH, Chen CC, Tseng PH, Liang JT, et al. Association between early stage colon neoplasms and falsenegative results from the fecal immunochemical test. Clin Gastroenterol Hepatol. 2013;11(7):832-8.e1-2. https://doi.org/10. 1016/j.cgh.2013.01.013.
27. Wong MC, Ching JY, Chan VC, Lam TY, Shum JP, Luk AK, et al. Diagnostic accuracy of a qualitative fecal immunochemical test varies with location of neoplasia but not number of specimens. Clin Gastroenterol Hepatol. 2015;13(8):1472-9. https:// doi.org/10.1016/j.cgh.2015.02.021.

28. Niedermaier T, Weigl K, Hoffmeister M, Brenner H. Diagnostic performance of flexible sigmoidoscopy combined with fecal immunochemical test in colorectal cancer screening: meta-analysis and modeling. Eur J Epidemiol. 2017;32(6):481-93. https:// doi.org/10.1007/s10654-017-0279-2.

29. Krebsneuerkrankungen (Inzidenz), altersstandardisierte Rate [Europastandard] (je 100.000 Einwohner). In: Tabellen zum GEKID-Atlas. Gesellschaft der epidemiologischen Krebsregister in Deutschland e.V. (GEKID). 2018. http://www.gekid.de/Atlas/ Tabellen/Tabellen_D.php?Method=INCIDENCE_EU\&ICD10= C18-C21\&Year_from $=2005 \&$ Year_to $=2013 \&$ Men $=$ on $\&$ Women $=$ on $\&$ Rates $=$ on. Accessed 6 March 2018.

30. Niedermaier T, Weigl K, Hoffmeister M, Brenner H. Diagnostic performance of one-off flexible sigmoidoscopy with fecal immunochemical testing in a large screening population. Epidemiology. 2018;29(3):397-406. https://doi.org/10.1097/EDE. 0000000000000795.

31. Sajid MS, Caswell JF, Abbas MA, Baig MK, McFall MR. Improving the view during flexible sigmoidoscopy: a systematic review of published randomized, controlled trials comparing the use of oral bowel preparation versus enema bowel preparation. Updates Surg. 2015;67(3):247-56. https://doi.org/10.1007/ s13304-015-0295-2.

32. Bibbins-Domingo K, Grossman DC, Curry SJ, Davidson KW, Epling JW Jr, Garcia FA, et al. Screening for colorectal cancer: US Preventive Services Task Force recommendation statement. JAMA. 2016;315(23):2564-75. https://doi.org/10.1001/jama. 2016.5989.

33. Schoenfeld P, Lipscomb S, Crook J, Dominguez J, Butler J, Holmes L, et al. Accuracy of polyp detection by gastroenterologists and nurse endoscopists during flexible sigmoidoscopy: a randomized trial. Gastroenterology. 1999;117(2):312-8.

34. Cooper MA, Tinmouth JM, Rabeneck L. Registered nurse-performed flexible sigmoidoscopy in Ontario: development and implementaton of the curriculum and program. Can J Gastroenterol Hepatol. 2014;28(1):13-8.

35. American Society for Gatrointestinal Endoscopy. Endoscopic Procedures. In: Newsroom. 2014. https://www.asge.org/home/ about-asge/newsroom/media-backgrounders-detail/endoscopicprocedures\#sigmoidoscopy. Accessed Jan 42018.

36. Brenner H, Niedermaier T, Chen H. Strong subsite-specific variation in detecting advanced adenomas by fecal immunochemical testing for hemoglobin. Int $J$ Cancer. 2017;140(9):2015-22. https://doi.org/10.1002/ijc.30629. 